\title{
Circadian Rhythm of Brain Activity Estimated using Visual Synchronization Tasks
}

\author{
Relations between brain activity and living activity
}

\author{
Kyota Aoki \\ Department of Information Systems Science, Graduate School of Engineering, Utsunomiya University \\ 7-1-2, Yoto, Utsunomiya, JAPAN \\ $+81-28-689-6247$ \\ kyota@is.utsunomiya-u.ac.jp
}

\begin{abstract}
The authors proposed the method to estimate the performance of motion control function with a visual synchronization task. The proposed method is enable to measure and estimate the motor control function precisely and easily. This paper confirms the ability of the proposed method to observe the circadian rhythm of a brain activity of a human. This paper shows the proposed method and the measuring process. Then, the experiments are shows and are discussed. The experiments include 165 trials. There are objective differences of the performances of a brain function among time zones. T-test confirms this result.
\end{abstract}

\section{CCS-Concepts}

Applied computing $\rightarrow$ Life and medical sciences $\rightarrow$ Consumer health

\section{Keywords}

circadian rhythm; brain activity; visual synchronization task; hands' movements; motor control function

\section{INTRODUCTION}

Circadian rhythm controls many functions of our body. From the red blood cells to body temperature [1] [2]. There are circadian rhythms in neuron cells. Our body shows circadian rhythm also. Our brain also a major part of our body to show circadian rhythm. A sleep is a main result of our body's circadian rhythm. With our activity, our brain performance changes. Our proposed visual synchronization task and measuring method is more precise than classical tapping test and Padua peg board test [1] [3]. Our proposed test is easy to execute in a short time. We will confirm the ability of the proposed visual synchronization task for measuring the circadian rhythm of our brain activity.

There are many motor tasks that measure the abilities of motor control functions of a human. They are the Purdue pegboard task,

Permission to make digital or hard copies of all or part of this work for personal or classroom use is granted without fee provided that copies are notmade or distributed for profit or commercial advantage and that copies bear this notice and the full citation on the first page. Copyrights for components of this work owned by others than ACM must be honored. Abstracting with credit is permitted. To copy otherwise, or republish, to post on servers or to redistribute to lists, requires prior specific permission and/or a fee. Request permissions from Permissions@acm.org.

$I C B C B$ '17, January 06-08, 2017, Hong Kong, Hong Kong

(C) 2017 ACM. ISBN 978-1-4503-4827-0/17/01 ..\$15.00

DOI: http://dx.doi.org/10.1145/3035012.3035028 a seal affixation task, a tray carrying task, etc. [4][5][6]. These tasks estimate the ability of a motor control function of a human based on the results of the tasks. There is no observation of the process of the tasks.

There are also some synchronization tasks used to measure motor control function of a human. One example is a synchronization of finger taps with periodically flashing visual stimuli and synchronization with an auditory metronome. In these tasks, the timing between the stimuli and the tapping is measured. There is no observation of the process of the tapping [7] [8] [9] [10] [11] [12] [13].

Recently, many cheap and easy measurement methods for the movements of a human body have been developed. For instance, some of these sensors include Kinect sensor, and Leap motion sensor [14] [15]. There are many applications that use those sensors for controlling computers. For instance, there are many video games that use those sensors for controlling an avatar in the games [16].

Using the new motion sensor, we can measure the motion of hands easily and precisely. The human hands are the parts of a body that can make the most complex movements. We have proposed a method that measures the precise movements of hands synchronizing the movements of hands on a display. The synchronization needs visual perception of the displayed hands' images and precise control of the arm muscles. The resulting measure is very sensitive. With this measure, we can observe the performance of the motor control function precisely.

This paper confirms the ability of the proposed method to measure the circadian rhythm of a brain activity of a human. This paper shows the proposed method and the measuring process. Then, the experiments are shown and discussed.

First, this paper proposes a task to synchronize hands' movements with visual presentation. Then, the author discusses the measure of motor control function about visual synchronization of hands' motions. Next, this paper discusses the experiments to measure the circadian rhythm and the experimental results. And last, the author concludes this work.

\section{VISUAL SYNCHRONIZATION TASK}

\subsection{Task}

There are many motor tasks that intend to measure the motor control function of a human. However, most of these tasks measure the results of the tasks. There are some tasks that measure the synchronization between a finger tap and stimuli. With human observations, it is difficult to measure the process of 
synchronizing movements. Now, we can use a Kinect sensor and a Leap Motion sensor. These sensors measure the three-dimensional movements of a human body. With these sensors, we can measure the precise movements of a human body.

We can synchronize our movements with each other. For instance, in playing a dance, dancers can synchronize their movements with each other. A synchronization of movement is more difficult work than a simple imitation of movement. To generate synchronized movements, we need to observe the motion to be synchronized. We need to generate the motion to be similar to the motion synchronized. We need to observe the generated motion synchronizing the original motion. We need to estimate the divergence between the original motion synchronized and the motion synchronizing the original motion. We need to control the speed of the motion synchronizing. These functions make the feedback loop. However, for compensating our brain's processing delay, we need to estimate the delay itself and to make feedforward. To make feedforward, we need to remember and to forecast the periodic motion.

This processing loop is shown in Figure 1. For estimating the total brain function, we need to include all the functions of a brain. The visual synchronization task includes vision and motor control functions. The vision includes not only the static sight, but also the dynamic sight.

The visual synchronization is more difficult than audio synchronization. So, we observe the wider brain functions with the visual synchronization tasks than the audio synchronization tasks.

Our proposed visual synchronization task is the synchronization between the position of stimuli on a display and the position of the hands. Our synchronization task is not the synchronization between the timing of the stimuli and the timing of action. The measurement of timing is only one scalar value. In our proposed synchronization task, the measurements of positions both of the stimuli and the hands are the sequence of a triple of the positions of the stimuli and the ones of both hands. In one cycle of movement, we have larger amount of measurements from the proposed visual synchronization task than the tapping test.

\subsection{Motor control function measure}

We have large amount of measurements in a trial. We hope to show a simple scalar measure that represents the total brain activity. We define the motor control function measure using FFT (Fast Fourier Transform) results of the measured poses of both hands in each cycle. If a subject makes complete synchronization to the stimuli, the resulting pose of both hands follow a complete sine curve. As a result, at every cycle of the rotation of hands, the result of FFT has a zero value at the second term or higher terms. We define the measure as (1). This measure increases with the amount of the difference of a hand's pose from ideal sine curve.

$$
N S M=\left(\sum_{x=2}^{t / 4} m_{x}\right) / m_{1}
$$

In (1), $t$ is the number of terms. $m_{x}$ is the absolute value of the $\mathrm{x}$ th term of the result of FFT. $m_{1}$ is the power of the lowest frequency. This represents a one cycle of a hand's rotation. If the rotation of a hand follows the stimuli images precisely, the $m_{1}$ carries all powers of the hand's rotation. Other terms carry no power. In the case, the measure in (1) is 0 .

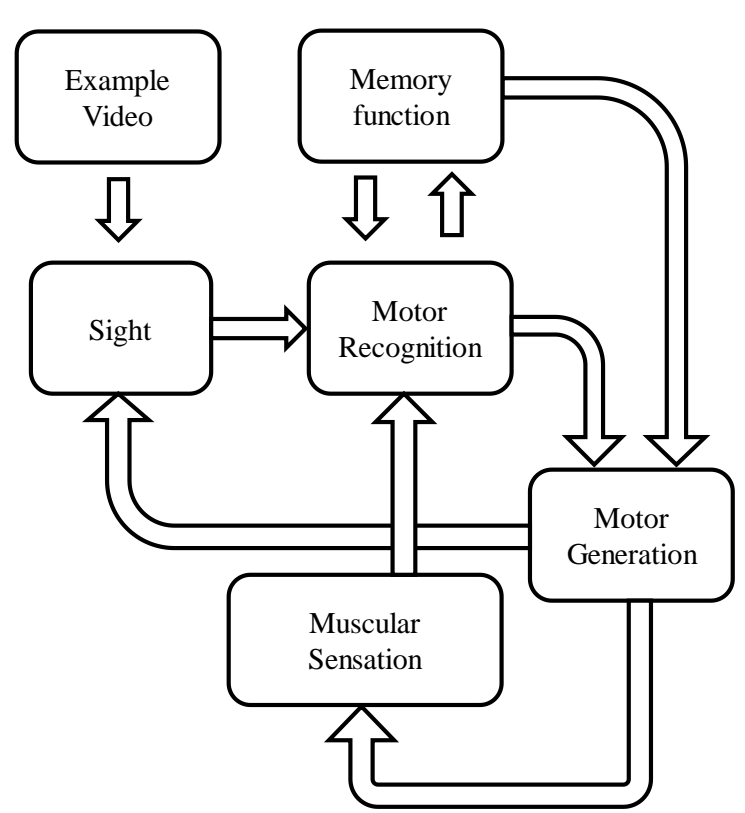

Figure. 1. Relation among functions.

$m_{0}$ is a value that represents the average of poses. This is not included in (1). As a result, this measure does not depend the absolute poses of hands.

We call this measure as Non-Smoothness-Measure (NSM). This measure may span from 0 to infinite. Our proposed system observes two hands. So at every cycle, we have two NSMs.

\section{MEASUREMENTS OF CIRCADIAN RHYTHM}

\subsection{Experiments}

We made 165 measurements from $9 / 11 / 2015$ to $20 / 2 / 2016$. Table 1 shows the distributions of trials in time.

In mid-nights, there is a few trials. There is no trial at 18 nor 19 o'clock. It is difficult to measure at fixed time and with fixed interval.

Figure 2 illustrates the distributions. Of cause, there is a few trials in mid-nights.

\subsection{Trials}

A trial is a 15 cycles of hands rotations. Each rotation of hands takes one second. There is 15 seconds at each trial. This is long enough to measure and easy to be done.

\subsection{Performance measure}

We have 15 pairs of NSMs at each trial. There are 30 NSMs. However, we hope to define a scalar measure that represents the brain activity of a subject from the 30 NSMs.

Our previous experiments show that the difference between a left hand and a right hand is small. However, we treat both hands. There is 15 NSMs at a hand. We can use the mean to define the scalar measure of a brain activity. However, it is difficult to keep the hands movements in 15 seconds. There is a difference among 30 NSMs. We can use the mean of all 30 NSMs. However, there 
are some error measurements. Our experiments show that the first cycle shows big NSMs, because a subject try to synchronize his hands' movements to the displayed hands' movements. There is a small NSM caused from accidentally matching between the displayed movement and the subject's movement.

We define the NSM of a trial as (1). We call the NSM of a trial as TNSM in the following.

TNSM =

$\min _{i=1,13, h=\{l, r\}} \operatorname{average}\left(N S M_{h, i}, N S M_{h, i+1}, N S M_{h, i+2}\right)$

In (2), $N S M_{h, i}$ is a $N S M$ of a hand $h$ at cycle $i$. The TNSM shows the best performance of a subject in a trial. We use this TNSM for evaluating the performance of a subject in a trial. In simply, the TNSM is the best performance of a subject in a trial. There are persons that can only keep their hands movements properly in a very short period. Those persons may have some kinds of problems about their brain functions. However, we only treat a brain performance in a short period.

\section{Results}

Table 2 shows the mean and the standard derivation (STD) of TNSMs in each time zone. A time zone starts from the just the hour and end to the next hour. The mean of TNSMs is 0.249 . The STD is 0.0503. Figure 3 shows the changes of the mean and STD. The bars represent the means. The lines show the STDs.

From Figure 3, it is difficult to find apparent patterns. We find three minimal time zones. They are 7,12 , and 15 o'clock. Our first question is "Really, they are minimal points?". We check the difference with the t-test.

\subsection{7 o'clock}

Table 3 shows the results of t-test between the time zone of 7 o'clock and ones of the following time zones. The mean of 7 o'clock is near the mean of 8 . The t-test between 7 and 8 o'clock says that the probability of the sameness is 0.39 . So, we cannot treat the TNSMs of 7 o'clock and the ones of 8 o'clock as different populations.

We treat the TNSMs both of 7 and 8 o'clock as a single population, and compare it with the TNSMs in other time zones. Table 4 shows the results of t-tests between time zone from 7 to 8 o'clock and other following time zones. The TNSMs of the time zone 7 and 8 o'clock shows difference of the TNSMs of time zone of 9 o'clock. The mean of TNSMs of 9 o'clock is apparently larger than the that of 7 and 8 o'clock.

\subsection{2 o'clock}

The mean of the TNSMs of the time zone of 12 o'clock is other least point. We show the result of t-test between the TNSs of 12 o'clock and ones of following time zones in Table 5.

Table 5 shows that the TNSMs of 12 o'clock is included in a population of 13 and 14 o'clock.
Table 1. Number of trials

\begin{tabular}{|c|c|}
\hline Time & Number of trials \\
\hline 0 & 0 \\
\hline 1 & 0 \\
\hline 2 & 3 \\
\hline 3 & 0 \\
\hline 4 & 0 \\
\hline 5 & 1 \\
\hline 6 & 19 \\
\hline 7 & 14 \\
\hline 8 & 7 \\
\hline 9 & 15 \\
\hline 10 & 10 \\
\hline 11 & 7 \\
\hline 12 & 5 \\
\hline 13 & 12 \\
\hline 14 & 14 \\
\hline 15 & 7 \\
\hline 16 & 9 \\
\hline 17 & 7 \\
\hline 18 & 0 \\
\hline 19 & 0 \\
\hline 20 & 9 \\
\hline 21 & 11 \\
\hline 22 & 11 \\
\hline 23 & 4 \\
\hline
\end{tabular}

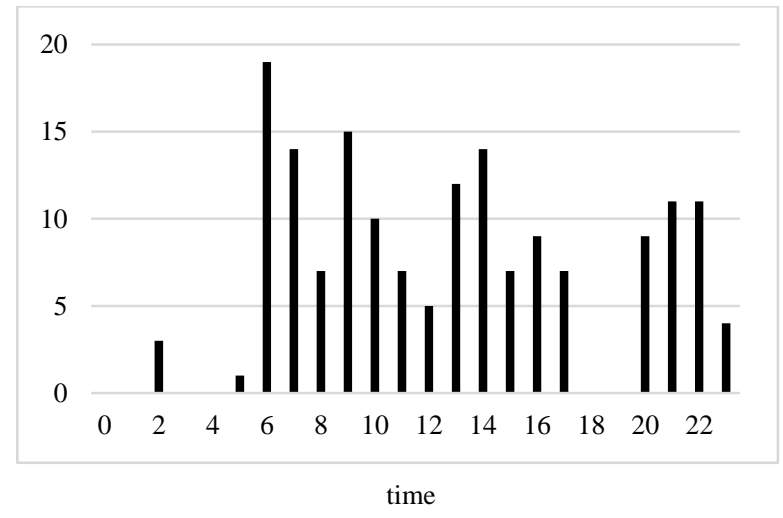

Figure 2. Distribution of the number of trials. 
Table 2. TNSMs

\begin{tabular}{|c|c|c|}
\hline Time & Mean & STD \\
\hline \multicolumn{3}{|l|}{0} \\
\hline \multicolumn{3}{|l|}{1} \\
\hline 2 & 0.251 & 0.0303 \\
\hline \multicolumn{3}{|l|}{3} \\
\hline \multicolumn{3}{|l|}{4} \\
\hline 5 & 0.232 & \\
\hline 6 & 0.260 & 0.0552 \\
\hline 7 & 0.220 & 0.0401 \\
\hline 8 & 0.225 & 0.0408 \\
\hline 9 & 0.260 & 0.0522 \\
\hline 10 & 0.246 & 0.0466 \\
\hline 11 & 0.241 & 0.0618 \\
\hline 12 & 0.215 & 0.0370 \\
\hline 13 & 0.242 & 0.0588 \\
\hline 14 & 0.252 & 0.0538 \\
\hline 15 & 0.210 & 0.0203 \\
\hline 16 & 0.251 & 0.0435 \\
\hline 17 & 0.263 & 0.0428 \\
\hline \multicolumn{3}{|l|}{18} \\
\hline \multicolumn{3}{|l|}{19} \\
\hline 20 & 0.267 & 0.0250 \\
\hline 21 & 0.282 & 0.0551 \\
\hline 22 & 0.262 & 0.0560 \\
\hline 23 & 0.229 & 0.0616 \\
\hline Total & 0.249 & 0.0503 \\
\hline
\end{tabular}

Table 3. Result of t-test of 7 o'clock

\begin{tabular}{|c|c|c|}
\hline o'clock & $\mathrm{t}$ & two-tailed test $p$-value \\
\hline 8 & -0.270 & 0.792 \\
\hline 9 & -2.276 & 0.032 \\
\hline 10 & -1.392 & 0.181 \\
\hline 11 & -0.753 & 0.476 \\
\hline
\end{tabular}

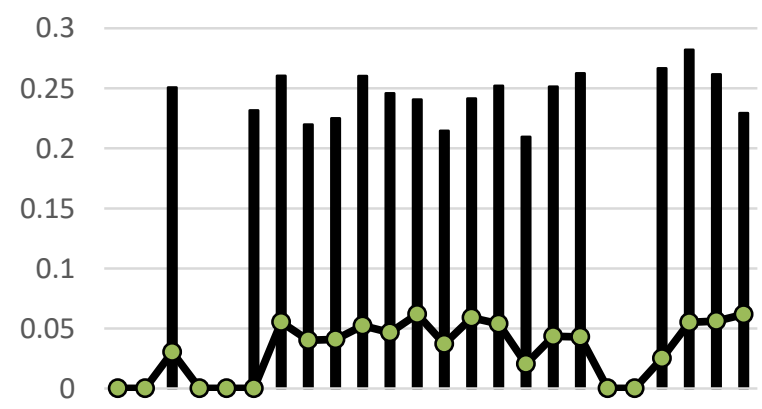

$\begin{array}{llllllllllll}0 & 2 & 4 & 6 & 8 & 10 & 12 & 14 & 16 & 18 & 20 & 22\end{array}$

Figure 3. The means and the STDs in time zines.
Table 4. Results of t-test of 7 and 8 o'clock

\begin{tabular}{|c|c|c|}
\hline o'clock & $\mathrm{t}$ & two-tailed test $p$-value \\
\hline 9 & -2.370 & 0.026 \\
\hline 10 & -1.399 & 0.181 \\
\hline 11 & -0.708 & 0.505 \\
\hline
\end{tabular}

Table 5. Results of t-test of 12 o'clock

\begin{tabular}{|c|c|c|}
\hline o'clock & $\mathrm{t}$ & two-tailed test $p$-value \\
\hline 13 & -1.135 & 0.278 \\
\hline 14 & -1.708 & 0.118 \\
\hline
\end{tabular}

Table 7. Subject's custom

\begin{tabular}{|c|c|}
\hline Time zone & Activity \\
\hline 6 & Wake up \\
\hline 7 & Breakfast \\
\hline 7,8 & Driving a car about 40 minutes. \\
\hline 12 & Lunch \\
\hline 16,17 & Driving a car about 40 minutes. \\
\hline 18 & Dinner \\
\hline
\end{tabular}

Then, he works. Around noon, he has a lunch, then works. Next, around 17 he drives a car to the home. Then, he has a dinner in 18 o'clock.

\subsection{Relation between activity and TNSMs}

After every ingestion, TNSMs increase in 2 hours. After ingestion, we may be sleepy. The measured TNSMs may represent this tendency objectively and numerically. The other effective activity is driving a car. After the time zone where a subject drives a car, there is a sharp increase of the TNSMs. This represent the tiredness of a subject. In driving a car, we must keep our attention.

\section{CONCLUSION}

This paper presents the ability of our proposed visual synchronization task and observation method that measures the motor control function of our brain. A subject shows a dairy change of the performance of motor control function. There are differences of the performances among time zones. This is confirmed by t-tests. However, this observed daily change may be a result of circadian rhythm or not is a problem.

Our measuring method of motor control function cannot distinguish the causes. We need to much more experiments with the precise life activity records.

Table 6. Results of t-test of 15 o'clock

\begin{tabular}{|c|c|c|}
\hline o'clock & $\mathrm{t}$ & two-tailed test $p$-value \\
\hline 13 & -1.715 & 0.107 \\
\hline 14 & -2.606 & 0.018 \\
\hline 16 & -2.432 & 0.035 \\
\hline 17 & -2.772 & 0.028 \\
\hline 20 & -5.047 & 0.000 \\
\hline 21 & -3.967 & 0.001 \\
\hline 22 & -3.967 & 0.001 \\
\hline 23 & -0.622 & 0.578 \\
\hline
\end{tabular}




\section{ACKNOWLEDGMENTS}

This work is supported with JSPS16K01057 and JSPS16K04818.

\section{REFERENCES}

[1] Xiaoxiao Qian, Susanne K. Droste, Stafford L. Lightman, Johannes M. H. M. Reul, and Astrid C. E. Linthorst, "Circadian and Ultradian Rhythms of Free Glucocorticoid Hormone Are Highly Synchronized between the Blood, the Subcutaneous Tissue, and the Brain," DOI=http://dx.doi.org/10.1210/en.2012-1484, July 2012.

[2] Li, Jun Z., et al. "Circadian patterns of gene expression in the human brain and disruption in major depressive disorder." Proceedings of the National Academy of Sciences, 110, 24, pp. 9950-9955, April 2013.

[3] Kyota Aoki, Hisanori Hotta, "Brain Activity Estimation with Precise Motor Measurements of Visual Synchronization Task of Hands", 2015, Global Health 2015, pp.39-44, ISBN: 9781-61208-424-4, ISSN: 2308-4553, Nice, France, July 2015.

[4] Lafayette Instrument, "Purdue Pegboard Test", http://www.lafayetteevaluation.com/product_detail.asp?ItemI $\mathrm{D}=159$, retrieved July 2015 .

[5] Shogo Hirata, Yoshio Kitajima, Tomio Hosobuchi, Mitsuru Kokubun, "The speed and accuracy of fine motor actions in children with intellectual disabilities," Bulletin of Tokyo Gakugei University, 59, pp. 263-267, 2008.

[6] Mitsuru Kokubun, "ARE CHILDREN WITH DOWN SYNDROME CAREFUL IN PERFORMING A TRAYCARRYING TASK THAN CHILDREN WITH OTHER TYPES OF MENTAL RETARDATION?" Perceptual and Motor Skills, 88:3c, pp. 1173-1176, 1999.

[7] Michael J. Hove, John R. Iversen, Allen Zhang, Bruno H. Repp, "Synchronization with competing visual and auditory rhythms:bouncing ball meets metronome," Psychological Research, 77, pp. 388-398, 2013.
[8] Yoshimori Sugano, Mirjam Keetels, Jean Vroomen, "The Build-Up and Transfer of Sensorimotor Temporal Recalibration Measured via a Synchronization Task," Front Psychol. 2012; 3: 246, doi:10.3389/fpsyg.2012.00246, 2012.

[9] Vanessa Krause, Bettina Pollok, Alfons Schnitzler, "Perception in action: The impact of sensory information on sensorimotor synchronization in musicians and nonmusicians," Acta Psychologica, Volume 133, Issue 1, pp. 28 37, January 2010.

[10] Jehee Lee, Jinxiang Chai, Paul S. A. Reitsma, "Interactive Control of Avatars Animated with Human Motion Data," ACM Transactions on Graphics (TOG). ACM pp. 491-500, 2002.

[11] Sarah Vercruysse, et al, "Freezing in Parkinson's disease: a spatiotemporal motor disorder beyond gait," Movement Disorders, 27(2), pp. 254-263, 2012 [14]Microsoft, "Kinect for Windows," https://www.microsoft.com/enus/kinectforwindows/, retrieved 2015/0707.

[12] Maurice A. Smith, Jason Brandt, Reza Shadmehr, "Motor disorder in Huntington's disease begins as a dysfunction in error feedback control," Nature, 403, pp. 544-549, February 2000.

[13] Katya Rubia, Anna B. Smith, Michael J. Brammer, Brian Toone, Eric Taylor, "Abnormal Brain Activation During Inhibition and Error Detection in Medication-Naïve Adolescents with ADHD," Am Psychiatric Assocvolume, 162, 6, pp. 1067-1075, June 2005.

[14] Sarah Vercruysse, et al, "Freezing in Parkinson's disease: a spatiotemporal motor disorder beyond gait," Movement Disorders, 27(2), pp. 254-263, 2012

[15] LEAP MOTION, "Leap Motion for Mac and PC," https://www.leapmotion.com/product/desktop, retrieved 2016/08/23.

[16] Microsoft, "Kinect for Xbox One", http://www.xbox.com/enUS/xbox-one/accessories/kinect, retrieved 2016/08/23. 